\title{
UMA INTRODUÇÃO AOS GRUPOS SOLÚVEIS
}

\section{AN INTRODUCTION TO SOLUBLE GROUPS}

Luís Henrique Gabriel de Macedo, Antônio Carlos Tamarrozzi, Roberto Junior Dias, Ana Paula Souza Santos

Universidade Federal do Mato Grosso do Sul. Grupo PET Matemática, Matemática-Três Lagoas luish.g.macedo@gmail.com

RESUMO - A teoria dos Grupos ocupa uma parte importante da Álgebra abstrata, sendo sua origem advinda do estudo das equações algébricas. Neste processo, a resolução de equações por meio de radicais está relacionada ao conceito de solubilidade de certos grupos associados. Por outro lado, o conceito de solubilidade tem interesse próprio, constituindo-se uma medida da comutatividade de um grupo. Neste trabalho desenvolvemos o conceito introdutório de grupos solúveis e exploramos algumas propriedades associadas. $O$ trabalho é um dos resultados de projetos de iniciação científica do Programa de Educação Tutorial (PET), vinculado ao curso de Matemática da UFMS. Campus de Três Lagoas. Nos projetos de iniciação científica, os alunos do PET, exploram tópicos avançados da Matemática que objetivam a iniciação à pesquisa, como também o desenvolvimento do tema como contribuição à formação dos demais alunos da graduação na área.

Palavras-chave: grupos quocientes; equações algébricas; série subnormal.

Recebido em: $x x / x x / x x x$ Revisado em: $x x / x x / x x x$ Aprovado em: $x x / x x / x x x$
ABSTRACT - Group theory occupies an important part of abstract algebra, its origin coming from the study of algebraic equations. In this process, the resolution of equations by means of radicals is related to the concept of solubility of certain associated groups. On the other hand, the concept of solubility has its own interest, constituting a measure of the commutativity of a group. In this work we develop the introductory concept of soluble groups and explore some associated properties. The work is one of the results of scientific initiation projects of the Tutorial Education Program (PET), linked to the UFMS Mathematics course. Campus of Três Lagoas. In the projects of scientific initiation, students of the PET, explore advanced topics of Mathematics that aim at the initiation to the research, as well as the development of the subject as contribution to the formation of the other students of the graduation in the area.

Keywords: quotient groups; algebraic equations; subnormal series. 


\section{INTRODUÇÃO}

O estudo de equações algébricas é um dos principais tópicos de álgebra, sendo a origem da estrutura algébrica grupo relacionada ao estudo da solubilidade de equações de grau superior a 5 por meio de radicais, que posteriormente foi mostrado impossível de se generalizar. A estrutura de grupo desempenha um papel fundamental nesse processo, dado que o matemático francês Évariste Galois (1811-1832) vinculou a resolução de equações por meio de radicais à solubilidade de um determinado grupo, o grupo de Galois.

Galois desenvolveu uma nova teoria que, não apenas provou a insolubilidade por radicais das equações algébricas de grau maior ou igual a 5, mas introduziu a base da estrutura matemática hoje conhecida como Grupo. A Teoria dos Grupos, por sua vez é o alicerce da álgebra abstrata, responsável pelo desenvolvimento de muitas áreas, até mesmo fora da Matemática .

Este trabalho objetiva apresentar uma introdução a teoria dos grupos solúveis e algumas de suas propriedades. Este tema normalmente é estudado no inicio dos cursos de pós-graduação em Matemática, no entanto o tema foi explorado como complemento à formação algébrica dos alunos do curso de graduação em Matemática da UFMS.

\section{METODOLOGIA}

O desenvolvimento do tema foi baseado na sequencia normalmente adotada pela literatura do assunto, destacamos, por exemplo, as referências Garcia e Lequain (2010), Gonçalves (2015), Herstein (1970) e Domingues e lezz (2003).

O trabalho explora as técnicas convencionais da Teoria dos Grupos, em particular destacamos comutadores, subgrupos normais, subgrupos característicos, grupos quocientes e conjugações.

A definição de solubilidade foi introduzida mediante o conceito de cadeia subnormal, ou seja:

Definição: Um grupo $G$ diz-se solúvel se admite uma cadeia de subgrupos:

$$
\begin{aligned}
& \{1\}=G_{0} \subseteq G_{1} \subseteq \cdots \subseteq G_{n}=G \\
& \text { tal que, para todo } i=1, \ldots, n, \text { cada }
\end{aligned}
$$
subgrupo $G_{i-1}$ é normal em $G_{i}$ com grupo quociente $G_{i} / G_{i-1}$ cíclico. Uma cadeia de subgrupos de $G$ com esta propriedade chama-se uma série subnormal abeliana de $G$ e os quocientes respectivos chamam-se fatores da série.

\section{RESULTADOS}

Destacamos os seguintes resultados iniciais acerca da solubilidade de um grupo $G$ :

Proposição: Todo grupo abeliano é solúvel. 
De fato, a cadeia $\{1\} \subseteq G$ é uma série subnormal abeliana.

Proposição: Todo subgrupo de um grupo solúvel $G$ é solúvel.

Se $H$ é um subgrupo de $G$, então existe uma série subnormal abeliana para $H$, cujos termos são $G_{i} \cap H$.

Definição: Dados $H$ e $K$ subgrupos de um grupo $G$, definimos o subgrupo de comutadores [ $H, K]$ por $\left\{h k h^{-1} k^{-1} \mid h \in H, k\right.$ $\in K$,. Em particular, se $H=K=G$, o subgrupo $[G, G]$ é chamado de subgrupo dos comutadores, denotado por $G^{\prime}$ ou $G^{(1)}$. Definimos, recursivamente, $G^{(0)}=G, G^{(1)}=$ $[G, G]$ e $G^{(i+1)}=\left[G^{(i)}, G^{(i)}\right]$, ou seja, $G^{(i+1)}$ é o subgrupo dos comutadores do grupo $G^{(i)}$.

Proposição: Um grupo $G$ é solúvel se, $e$ somente se, $G^{(n)}=\{e\}$ para algum inteiro $n$ positivo

\section{Demonstração:}

Caso $G$ possua uma série de subgrupos

$$
\begin{aligned}
& \{e\}=G_{n} \unlhd G_{n-1} \unlhd \ldots \ldots . . . G_{1} \unlhd G \\
& \quad \operatorname{com} G_{i+1} \unlhd G_{i} \quad e^{G_{i}} / G_{i+1} \text { abeliano }
\end{aligned}
$$

$\forall i=0,1,2, \ldots ., n$.Sabemos que o grupo dos comutadores são o menor subgrupo com a propriedade de ser normal e que gera quocientes abelianos. Assim temos:

$$
G^{(i)} \leq G_{i} \forall i=0, \quad 1,2, \ldots ., n \text { e portanto }
$$
$G^{(n)} \leq G_{n}=\{e\}$, que leva a $G^{(n)}=\{e\}$, logo $G$ é solúvel.

Reciprocamente, se $G^{(n)}=\{e\}$ a série de comutadores:

$$
\{e\}=G^{n} \unlhd G^{n-1} \unlhd \ldots \ldots . . \unlhd G \unlhd G
$$

é uma série subnormal abeliana pois $G^{(i+1)} \unlhd$ $G^{(i)}$ e todo grupo quociente $\frac{G^{(i)}}{G^{(i+1)}}$ é abeliano.

Proposição: Seja G um grupo solúvel, então todo subgrupo e toda imagem homomorfica de $G$ são solúveis. Reciprocamente, se $N \unlhd G$ e $G / N$ são solúveis então $G$ é solúvel. Demonstração:

Seja $G$ solúvel, existe então $k$ tal que $G^{(k)}=\{e\}$. Se $H \leq G$, então $H^{(k)} \leq G^{(k)}=\{e\}$, ou seja $H^{(k)}=\{e\}$ e portanto $H$ é solúvel. Agora, seja $\varphi: G \rightarrow H$ um homomorfismo sobrejetivo. Dados $a, b \in G, \varphi\left(a b a^{-1} b^{-1}\right)=$ $\varphi(a) \varphi(b) \varphi(a)^{-1} \varphi(b)^{-1}$. Daí, $H^{\prime}=\varphi\left(G^{\prime}\right)$, e por indução, $H^{(n)}=\varphi\left(G^{(n)}\right)$. Portanto, $H^{(k)}=$ $\varphi\left(G^{(k)}\right)=\varphi(\{e\})=\{e\}$. Isto prova que toda imagem homomórfica de $G$ é solúvel. Reciprocamente, seja $N \unlhd G$ tal que $N$ e $G / N$ são solúveis. Então existem $k, l \in \mathbb{Z}$ tais que $N^{(k)}=\{e\}$ e $(G / N)^{(l)}=\{\bar{e}\}$. Como $G / N$ é a imagem homomórfica de $G$, pelo homomorfismo canônico $G \rightarrow G / N$, segue que $(G / N)^{(n)}=G^{(n)} N / N$ para todo inteiro positivo n. Daí, $G^{(l)} \leq N$. Portanto, $G^{(l+k)} \leq N^{(k)}$ $=\{e\}$ e $G$ é solúvel. 
Corolário: Sejam $H$ e $K$ grupos, então $H \times K$ é solúvel se, e somente se, $H$ e $K$ são solúveis.

\section{Demonstração:}

Se $H \times K$ é solúvel, então $H \simeq H \times\{e\}$ e $K \simeq\{e\} \times K$ são subgrupos normais. Logo, $H$ e $K$ são solúveis. Reciprocamente, suponha $H$ e $K$ são solúveis. Então $H \unlhd H \times K$ e $\frac{H \times K}{K}$ $\simeq K$ são solúveis, portanto $H \times K$ é solúvel.

Em se tratando da solubilidade de grupos finitos, apresentamos os seguintes resultados de impacto:

Teorema (Teorema de Burnside) Se G é um grupo com $|G|=p^{n} q^{m}$, p e $q$ números primos e $m, n \in \mathbb{N}$, então $G$ é solúvel.

Em geral não são validas generalizações de teoremas de Sylow para mais que um primo $p$. entretanto, essas generalizações são válidas para grupos abelianos e mais geralmente também para grupos solúveis assim:

Teorema: Seja G um grupo solúvel finito de ordem $a b \operatorname{com} \operatorname{mdc}(a, b)=1$. Então

1) Existe um subgrupo de $G$ de $\operatorname{ordem} a$;

2) Todos os subgrupos de ordem $a \operatorname{em~} G$ são conjugados;

3) Se $H$ é um subgrupo de $G$ tal que $|G| / a$, então existe um subgrupo $K$ de $G \operatorname{com}|K|=$ a tal que $H$ está contido em $K$.
Definição :Diremos que um grupo é supersolúvelse existir uma série

$$
\{e\}=G_{n} \unlhd G_{n-1} \unlhd \ldots \ldots . . \unlhd G_{1} \unlhd G
$$

Tal que $G_{i+1} \unlhd G i=0,1,2, \ldots . ., n$ com quocientes $G_{i} / G_{i+1}$ cíclicos.

Todo grupo supersolúvel é solúvel, mas reciproca não é verdadeira. Podemos tomar o exemplo de $A_{4}$, um grupo solúvel tal que $\{e\} \unlhd V \unlhd A_{4}$, onde $V$ é o grupo de Klein.

Proposição: Sejam $G, H, e, K$ grupos.

1. Se $G$ é supersolúvel, então todo subgrupo e todo quociente de $G$ é supersolúvel;

2. Se $H$ e $K$ são supersolúveis, então $H \times K$ é supersoúvel;

3. Se $G$ é supersolúvel finito e $N$ é normal minimal em $G$, então $|N|=p$;

\section{DISCUSSÃO}

A Teoria de Grupos possui várias aplicações em matemática pura, e tem se mostrado uma ferramenta útil a aplicações que tratam a física de partículas e física teórica. No caso dos grupos solúveis as proposições dadas são importantes ferramentas no estudo da teoria de Galois, pois com objetivo de estudar equações polinomiais surgiram as definição de grupos utilizadas. Dentro dessa teoria, cada polinômio pode ser associado a um grupo chamado grupo de Galois. Podemos dizer se 
este polinômio é solúvel por radicais se o grupo de Galois é solúvel.

Assim, o estudo de grupos solúveis pode ser feito por interesse puramente teórico, como também relacionado à teoria de Galois, como parte integrante dela.

\section{CONCLUSÃO}

No trabalho apresentamos um desenvolvimento inicial das propriedades e características dos Grupos Solúveis e observamos o envolvimento do tema com a resolução de equações algébricas. Por outro lado, em uma perspectiva puramente teórica, a solubilidade de Grupos tem interesse próprio, em se tratando de aproximar Grupos genéricos aos Grupos abelianos. São utilizadas técnicas e métodos convencionais da Teoria dos Grupos como normalidade, comutadores grupos quocientes e imagens homomórficas. O trabalho é concluído com a definição de grupos supersolúveis que é uma extensão da solubilidade.

\section{REFERÊNCIAS}

DOMINGUES, H. H.; IEZZI, G. Álgebra moderna. São Paulo: Atual, 2003.

GARCIA, A.; LEQUAIN, Y. Elementos de álgebra. 5. ed. Rio de Janeiro: IMPA, 2010.

GONÇALVES, A. Introdução a álgebra. 5. ed. Rio de Janeiro: IMPA, 2015
HERSTEIN, I. Tópicos de álgebra. São Paulo: Polígono, 1970. 